\section{Survey of use of anticoagulation in patients with atrial fibrillation}

\author{
Philip M W Bath, Abhiram Prasad, \\ Martin M Brown, Graham A MacGregor
}

Blood Pressure Unit, St George's Hospital Medical School, London SW17 0RE

Philip M W Bath, lecturer in medicine

Abhiram Prasad, medical registrar

Graham A MacGregor, professor of cardiovascular medicine

Division of Clinical Neuroscience, St George's Hospital Medical School, London SW17 0RE

Martin M Brown, senior

lecturer in neurology

Correspondence to: Dr P M W Bath

Department of Medicine, King's College School of Medicine and Dentistry, London SE5 9PJ.

BMF 1993;307:1045 had a specific contraindication.

\section{Patients, methods, and results} available.
Five randomised controlled trials show that warfarin reduces the risk of ischaemic strokes and death in patients with atrial fibrillation. ${ }^{1}$ We suspected that many such patients were not receiving warfarin and therefore reviewed the records of previous inpatients with atrial fibrillation to see how many were receiving warfarin or aspirin and whether those who were not

We performed a retrospective analysis of case notes of patients admitted from January 1991 to September 1992. The clinical coding department provided a list of 726 admissions (in random order) in which the patient was discharged with a diagnosis of atrial fibrillation; the first 100 notes were requested, of which 95 were

Fifty seven patients were drawn from general medical firms and the rest from the departments of cardiology (17), geriatrics (14), and surgery (seven). Forty eight were men. The median age was 79 (range 32-100). Nineteen patients had paroxysmal atrial fibrillation. The median duration of atrial fibrillation was 20 (4-226) months and it most commonly had an underlying cardiovascular cause (table).

Cause of atrial fibrillation in 95 patients

\begin{tabular}{lc}
\hline Cause & No of patients \\
\hline Ischaemic heart disease & 24 \\
Hypertension & 15 \\
Valve disease & 10 \\
Respiratory disease & 6 \\
Alcohol misuse & 2 \\
Cardiomyopathy & 2 \\
Postoperative complication & 2 \\
Thyrotoxicosis & 1 \\
Lone atrial fibrillation & 1 \\
Unknown & 32 \\
\hline
\end{tabular}

Seventy five patients were receiving antiarrhythmic treatment on discharge (digoxin (64), $\beta$ blocker (three), calcium antagonist (four), amiodarone (four)). Only 22 were taking warfarin while 16 were taking aspirin and one dipyridamole. Interestingly, aspirin had been prescribed for coexisting vascular disease rather than as prophylaxis against stroke. The notes of 20 patients showed a contraindication for anticoagulation: history of peptic ulcer disease or gastrointestinal bleeding (13), excessive alcohol intake (two), and dementia (two). Of the 75 patients without a documented contraindication, 36 (48\%, 95\% confidence interval $36 \%$ to $60 \%$ ) were receiving neither warfarin nor aspirin.
Overall, 22 of the 95 patients had suffered a stroke, of which 10 were ischaemic and one haemorrhagic; computed tomography had not been performed in 11 patients. Of the 10 patients with ischaemic stroke, three had had atrial fibrillation before their stroke and yet they had not been prescribed prophylactic warfarin or aspirin despite having no contraindication; one of these strokes was fatal. The patient who had a haemorrhagic stroke was not taking warfarin or aspirin. Five patients suffered a systemic embolic event (two brachial artery, two mesenteric artery, and one femoral artery). Two of them had had atrial fibrillation before the event and again had not been prescribed warfarin or aspirin despite having no contraindication.

\section{Comment}

This analysis shows that many patients with atrial fibrillation are not prescribed warfarin despite the absence of contraindications. This state of affairs is unlikely to be unique to this hospital and is also seen in general practice. ${ }^{2}$ This is surprising in view of the overwhelming evidence that warfarin leads to a profound reduction in the number of strokes. ${ }^{1}$ Five patients who had suffered a stroke or other systemic embolism after they had had atrial fibrillation had not been prescribed warfarin or aspirin despite there being no contraindication. The clinical trials suggest that three of these five events could have been prevented by anticoagulation. Clearly, our analysis does not allow us to comment on the risks of anticoagulation. The five published trials show, however, that the risk of bleeding is small. ${ }^{3}$

All patients with established atrial fibrillation, whether paroxysmal or not, should receive anticoagulants unless $(a)$ there is a specific contraindication, $(b)$ the patient is young with no obvious cause for the atrial fibrillation and therefore at low risk of stroke (lone atrial fibrillation), or (c) the patient is older than 80 , when the risks from anticoagulants are high. If warfarin is contraindicated and patients are less than 75 then aspirin ( $300 \mathrm{mg}$ per day) should be considered in the light of existing evidence. The forthcoming results of the European atrial fibrillation trial show that anticoagulation is also highly beneficial in the secondary prevention of stroke. Treatment with anticoagulants or aspirin seems to be cost effective provided that the risks of bleeding are kept low. ${ }^{4}$ Clearly, anticoagulation clinics will need large increases in funding to deal with the rising referral of patients with atrial fibrillation, or routine testing will need to be performed in the community.

1 Lowe GDO. Antithrombotic treatment and atrial fibrillation. BMf 1992;305: 1445-6.

2 Barnaby J, Howitt AJ. Antithrombotic treatment and atrial fibrillation. $B M \mathcal{F}$ 1993;306:207.

3 Ramsay LE. Warfarin in chronic atrial fibrillation. Lancet 1993;341:1376-7.

4 Gustafsson C, Asplund K, Britton M, Norrving B, Olsson B, Marke L-A. Cost effectiveness of primary stroke prevention in atrial fibrillation: Swedish national perspective. $B M \Im$ 1992;305:1457-60.

(Accepred 7 Fuly 1993)

\section{ONE HUNDRED YEARS AGO}

\section{NO BEDS}

This is the answer which has been commonly returned during the last fortnight to an application for the admission of a patient to a London hospital. At the present time they are as full as they will hold, and it has been practically impossible to obtain admission for half the serious cases which have stood in need of hospital treatment. This is true not only of the fever but also of the general hospitals, under which term we would include the children's hospitals. The extraordinary pressure is due to the coexistence of epidemics of typhoid fever and diphtheria, combined with the increasing prevalence of influenza, which has been noticed during the last three or four weeks in the East End of London, and has now extended to the West and South.

(BMF 1893;ii:1292.) 